\title{
Spin voltage generation through optical excitation of complementary spin populations
}

\author{
Federico Bottegoni ${ }^{1}$, Michele Celebrano ${ }^{1}$, Monica Bollani ${ }^{2}$, Paolo Biagioni ${ }^{1}$, Giovanni Isella ${ }^{1}$, \\ Franco Ciccacci ${ }^{1}$ and Marco Finazzi ${ }^{1 \star}$
}

By exploiting the spin degree of freedom of carriers inside electronic devices, spintronics has a huge potential for quantum computation and dissipationless interconnects ${ }^{1}$. Pure spin currents in spintronic devices should be driven by a spin voltage generator, able to drive the spin distribution out of equilibrium without inducing charge currents. Ideally, such a generator should operate at room temperature, be highly integrable with existing semiconductor technology, and not interfere with other spintronic building blocks that make use of ferromagnetic materials. Here we demonstrate a device that matches these requirements by realizing the spintronic equivalent of a photovoltaic generator. Whereas a photovoltaic generator spatially separates photoexcited electrons and holes, our device exploits circularly polarized light to produce two spatially well-defined electron populations with opposite in-plane spin projections. This is achieved by modulating the phase and amplitude of the light wavefronts entering a semiconductor (germanium) with a patterned metal overlayer (platinum). The resulting light diffraction pattern features a spatially modulated chirality inside the semiconductor, which locally excites spin-polarized electrons thanks to electric dipole selection rules ${ }^{2}$.

The integration of spintronics and photonics represents one of the most challenging tasks in modern optoelectronics ${ }^{3-7}$. In this context germanium plays a key role, owing to the large spin-orbit coupling and long electron spin lifetime $e^{8,9}$, integrability with Si-based electronic platforms, and optical properties matching the conventional telecommunications window ${ }^{10,11}$. In the optical orientation process ${ }^{2}$, dipole selection rules for optical transitions with circularly polarized light allow the excitation of an electron population in the Ge conduction band with a spin polarization $P=\left(n_{\uparrow}-n_{\downarrow}\right) /\left(n_{\uparrow}+n_{\downarrow}\right)$ up to $50 \%$ (refs $2,12,13$ ), where $n_{\uparrow(\downarrow)}$ are the up (down) spin densities (as referred to a proper quantization axis). This technique exploits the spin-orbit interaction, which allows the induction of a net spin polarization by manipulating the electron orbital degrees of freedom with circularly polarized light. The spinorbit interaction removes the energy degeneracy between heavy hole $(\mathrm{HH})$ and light hole $(\mathrm{LH})$ states and split off $(\mathrm{SO})$ states of the valence band at the $\Gamma$ point of the Ge Brillouin zone: a net electron spin polarization is obtained when the photon energy is tuned to the direct bandgap $E_{\mathrm{d}}$ (in $\mathrm{Ge} E_{\mathrm{d}} \approx 0.8 \mathrm{eV}$ at room temperature) and electrons coming only from $\mathrm{HH}$ and $\mathrm{LH}$ states are promoted to the conduction band. In Ge, the splitting $\Delta E_{\mathrm{SO}}$ between $\mathrm{HH}-\mathrm{LH}$ and SO states at $\Gamma$ is rather large, $\Delta E_{\mathrm{SO}} \approx 300 \mathrm{meV}$, whereas in $\mathrm{Si}$, for example, $\Delta E_{\mathrm{SO}}$ is only $40 \mathrm{meV}$, which represents a demanding constraint to avoid excitation of electrons from SO states. Indeed, although the first demonstration of optical orientation was done in $\mathrm{Si}$, only very small polarization values are achievable in such a material ${ }^{14}$, whereas a polarization comparable to those obtained in III-V semiconductors has been observed at the direct gap of $\mathrm{Ge}$ (refs 2,12,13)

The spin-oriented electrons excited at the bottom of the Ge conduction band at $\Gamma$ are scattered to the L minima within $\sim 300 \mathrm{fs}$, partially maintaining the initial spin orientation ${ }^{9}$. As the electron spin lifetime in bulk Ge is in the nanosecond range ${ }^{8}$, whereas the hole spin lifetime is much shorter (a few hundreds of femtosecond $\mathrm{s}^{15}$ ), spin transport phenomena in Ge can be assumed to be associated mainly with electrons.

Although optical orientation allows the generation of much higher spin polarizations in semiconductors as compared with all-electrical spin-injection schemes, only out-of-plane spin polarizations can be achieved at normal incidence: this limits the integration with spin-readout blocks, such as magnetic tunnel junctions, to those exploiting out-of-plane magnetization ${ }^{16}$. Illumination at grazing incidence can comply with in-plane magnetization devices ${ }^{17}$, although it does not represent a convenient geometry for optical orientation.

To drive a spin current into a device, one should be able to induce a spin voltage, which is a difference in the spin splitting of the electrochemical potential between two points in space. This can be generated by locally injecting (electrically, optically or thermally) an excess density of carriers with one spin orientation only. Spin diffusion will then produce a gradient in the spin splitting and thus a spin voltage between different locations. However, in general such a spin voltage would also be accompanied by the electron/hole chemical potential drop. In this respect, the creation of two spin populations with opposite sign would allow the generation of pure (that is, not accompanied by electron/hole chemical potential difference) spin currents, which would also be more intense and more localized than those achievable when only one population is generated.

To exploit the advantages of complementary spin populations, one would need to create them over distances shorter than the spin diffusion length. In this regard, we note that, thanks to the high refractive index of semiconductors (in Ge $n_{\mathrm{Ge}} \approx 4.3$ at $\lambda=1,550 \mathrm{~nm}$, corresponding to $h v \approx 0.8 \mathrm{eV}$ ), the light diffraction limit is much smaller than the spin diffusion length (which is about $2.5 \mu \mathrm{m}$ in room-temperature Ge). Therefore this task could be accomplished by optical orientation through an appropriate engineering of the light wavefront.

We have addressed these issues by patterning a thin overlayer onto the semiconductor surface to spatially modulate the phase and amplitude of the electromagnetic wavefronts on the semiconductor 
a

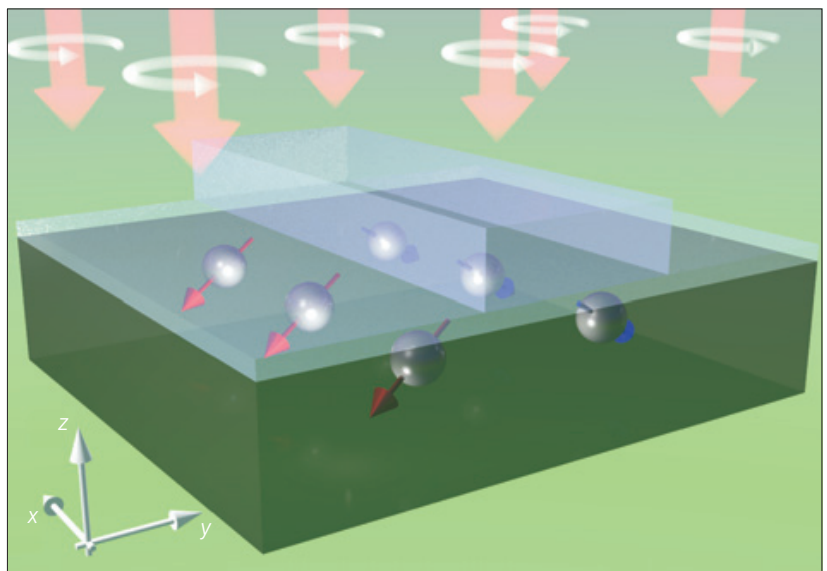

b
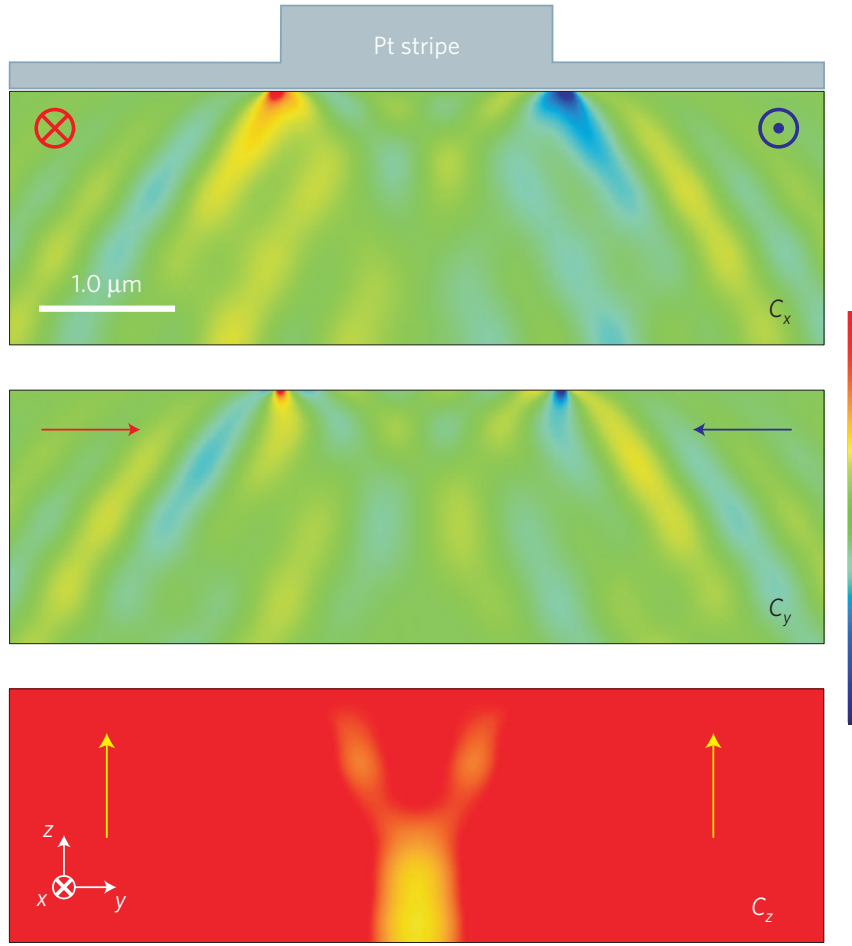

c

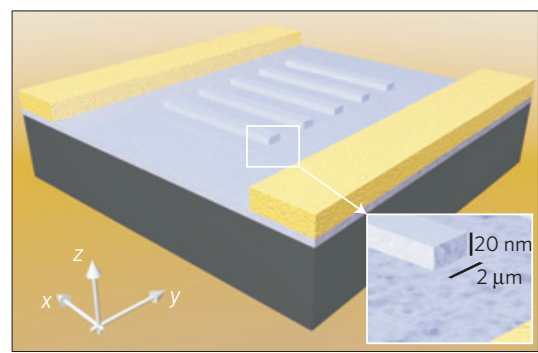

d
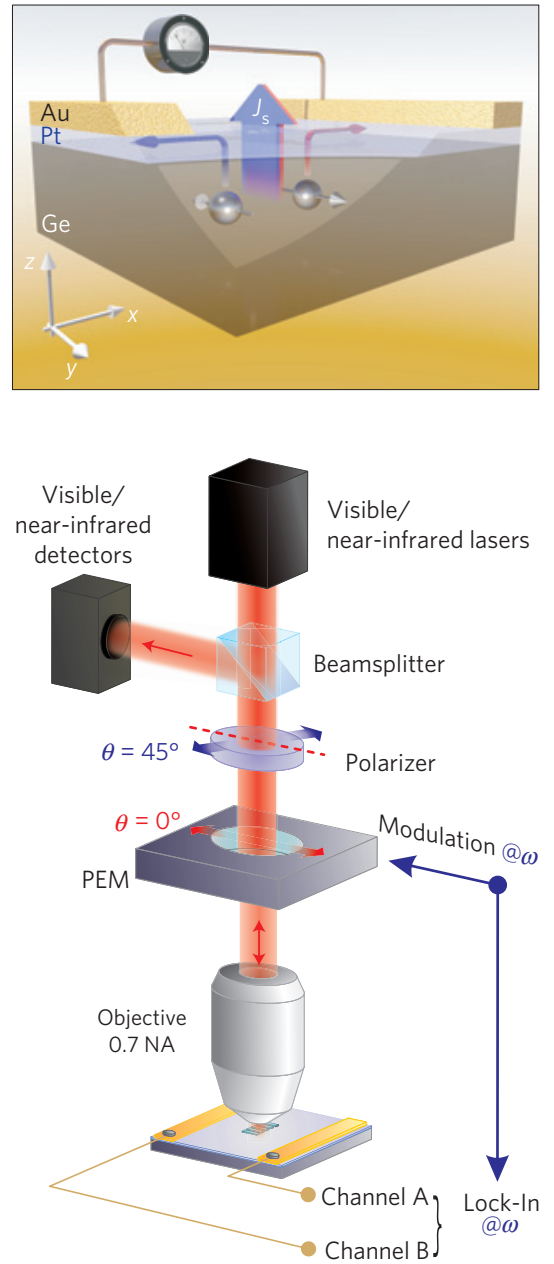

Figure 1 | Spin voltage generation and experimental set-up for spatially resolved inverse spin Hall effect (ISHE) measurements. a, Illustration of the working principle for spin voltage generation: when a Pt microstripe is placed on top of a Ge substrate, illumination at normal incidence ( $z$ axis) with right-circularly polarized light $(h v=0.8 \mathrm{eV})$ allows the simultaneous generation of two symmetric spin densities with opposite in-plane spin projections inside $\mathrm{Ge}$ in correspondence with the stripe edges. $\mathbf{b}$, The local spin density projected along the $x, y$ or $z$ axis of photogenerated electrons is proportional to the Stokes parameter $c_{x}=2 \operatorname{lm}\left(E_{z} E_{y}{ }^{*}\right), c_{y}=2 \operatorname{lm}\left(E_{x} E_{z}{ }^{*}\right)$ and $c_{z}=2 \operatorname{lm}\left(E_{y} E_{x}{ }^{*}\right)$, respectively, with $E_{x}, E_{y}$ and $E_{z}$ being the (complex) components of the electric field. c, Sketch of the sample geometry. $\mathbf{d}$, ISHE detection geometry in the investigated sample: the photo-generated electrons with a spin polarization $P_{y}$ yield a spin current density $J_{S}$ diffusing along the $z$ axis from $n$-doped $\mathrm{Ge}$ to the Pt layer, where the ISHE provides the conversion of $J_{S}$ into the electromotive

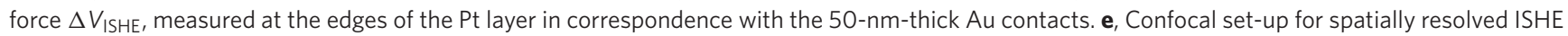
measurements: the collimated excitation beam enters the photo-elastic modulator (PEM), which modulates the light circular polarization at a frequency $\omega=50 \mathrm{kHz}$. The light beam is then focused by a microscope objective (numerical aperture, NA $=0.7$ ) on the Pt microstructures. While a raster scan of the sample is performed, the differential voltage signal $\Delta V_{\text {ISHE }}$ is detected by a lock-in amplifier demodulating at $\omega$.

sample at normal incidence. This method allows the creation of a field distribution inside the semiconductor with spatially engineered chirality with respect to an in-plane quantization axis (that is, in the direction perpendicular to the wavevector of the light). The circular polarization state of the light with respect to a Cartesian reference system $x, y, z$ is described by the Stokes parameters ${ }^{18} c_{x}=2 \operatorname{Im}\left(E_{z} E_{y}{ }^{*}\right), \quad c_{y}=2 \operatorname{Im}\left(E_{x} E_{z}{ }^{*}\right)$ and $c_{z}=2 \operatorname{Im}\left(E_{y} E_{x}{ }^{*}\right)$, with $E_{x}, E_{y}$ and $E_{z}$ being the (complex) components of the electric field inside the semiconductor along the $x, y$ and $z$ axes, respectively. The components of the spin polarization $\mathbf{P}$ resulting from optical orientation are proportional to the Stokes parameters evaluated along the corresponding axes. The simplest configuration allowing us to obtain sizeable $c_{x}$ and $c_{y}$ values consists of a metal stripe deposited on the semiconductor surface. Figure 1a presents a pictorial scheme of the investigated structure and Fig. 1b shows the distribution of the simulated Stokes 


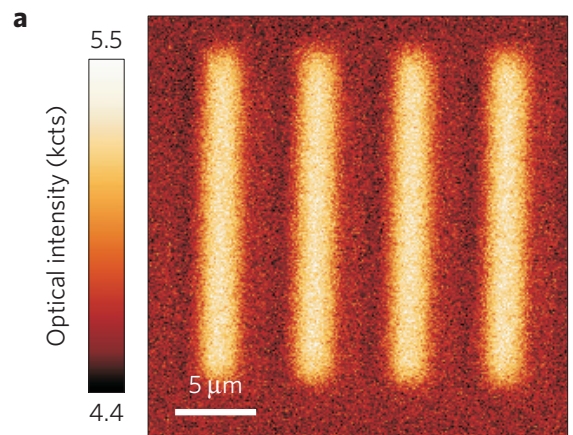

b

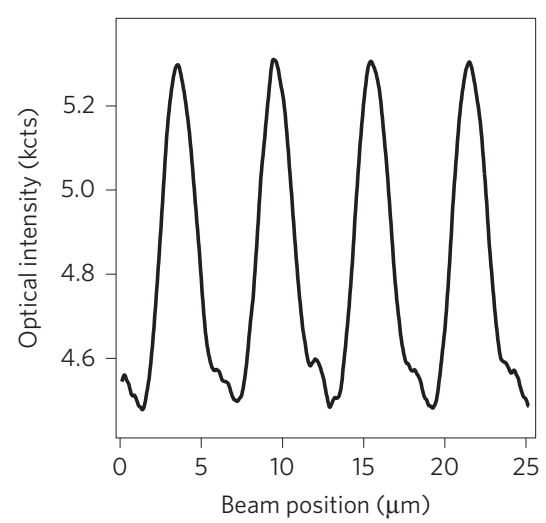

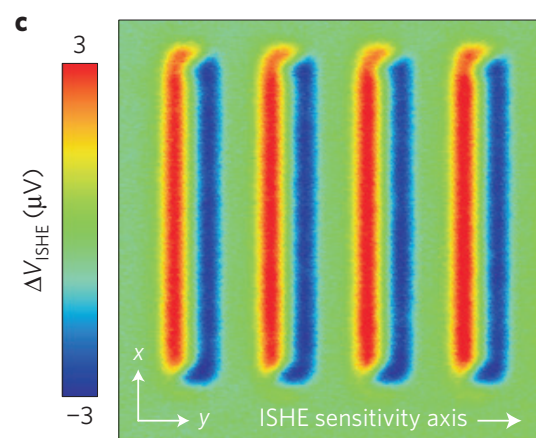

e

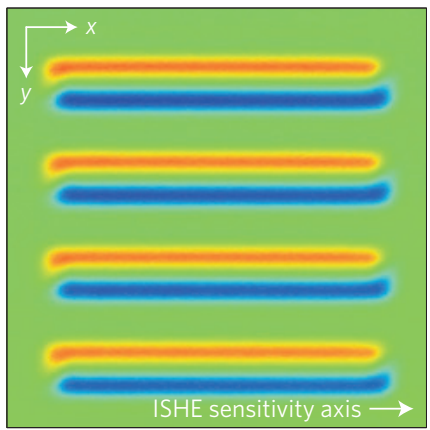

d

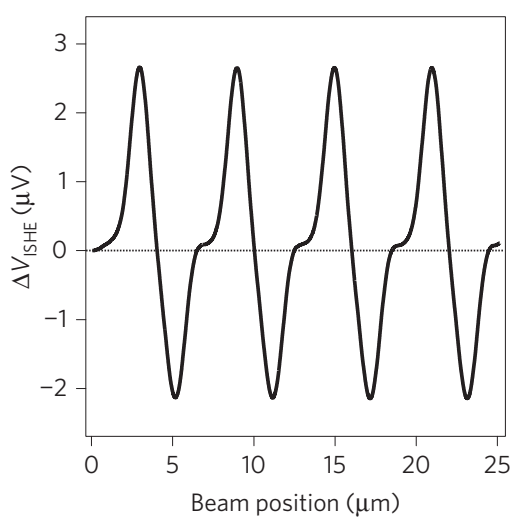

$\mathbf{f}$

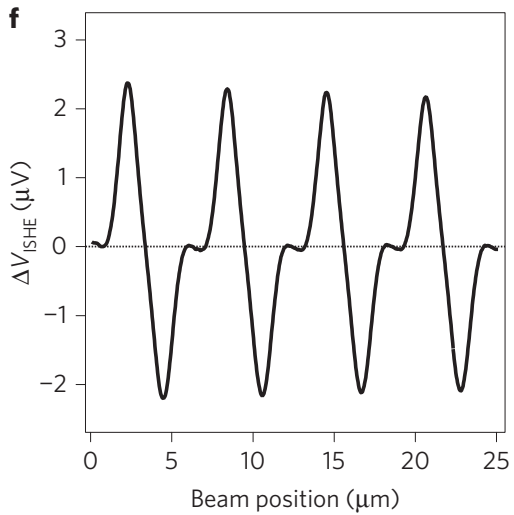

Figure 2 | Measurement of optical spin injection and detection through ISHE. a, 2D optical reflectivity map of the nanostructured Pt/n-doped Ge sample,

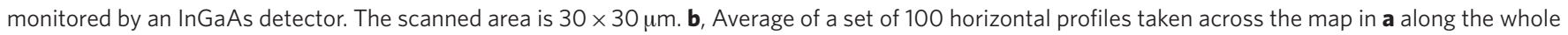

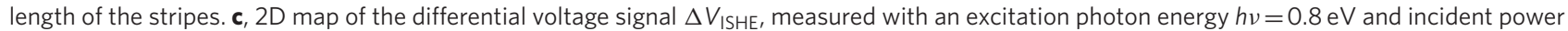
$W=150 \mu \mathrm{W}$. d, Average of a set of 100 horizontal profiles taken across the map in $\mathbf{c}$ along the whole length of the stripes. e, Same as $\mathbf{b}$, but obtained with

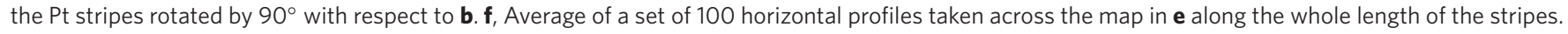

parameters inside Ge (see the Methods for details) when the stripe is illuminated at normal incidence with a circularly polarized plane wave and a photon energy resonant with the Ge direct band gap. The light scattering at the stripe edges creates a local field with $c_{x}$ (with $x$ parallel to the stripe long axis) and $c_{y}$ distributions (Fig. 1a) that excite two spatially separated populations of spin-oriented electrons with opposite spin projections in the sample plane. In contrast the simulated $c_{z}$ parameter shows that when $z$ (perpendicular to the sample surface) is chosen as the quantization axis, only one spin population is generated below the metal overlayer. Notably, the simulations of Fig. $1 \mathrm{~b}$ show that in the proximity of the stripe edges the absolute values of $c_{x}, c_{y}$ and $c_{z}$ are roughly equal, indicating that a significant in-plane spin polarization projection is generated immediately underneath the metal layer, with an angle between the averaged spin polarization vector and the plane of the device below $60^{\circ}$. This effect does not depend on the particular choice of the semiconductor/patterning material combination, but rather on the geometry of the patterning and on the dipole selection rules for the semiconductor. Notably, this concept can be confined to sub-micrometer scales (see below) and extended to a wide range of architectures and different semiconductor/patterning material combinations. Moreover, we stress that, at variance with the spin solar cell reported in ref. 4 , where a net charge current is obtained together with spin diffusion, our device generates a pure spin electrochemical potential drop inside the semiconductor that is totally decoupled from any charge separation. In fact, possible charge currents induced by photoelectric effects are sensitive only to the intensity of light and not to its polarization state. As the stripes are symmetric, there cannot be any electric potential difference across them. In perspective, this could allow, together with the submicrometric control and manipulation of the spin dynamics, the exploitation of the dissipationless features provided by pure spin transport. For all the reasons listed above, and thanks to the easy integrability with current semiconductor technology, we believe that the proposed architecture could represent an important complementary device adding new functionalities to the palette of tools available at present for spintronic applications.

The device described in Fig. 1a has been realized by uniformly evaporating a 4-nm-thick Pt layer on top of an As-doped Ge substrate $\left(5 \times 5 \mathrm{~mm}^{2}, N_{\mathrm{d}}=2 \times 10^{16} \mathrm{~cm}^{-3}\right)$. A set of Pt stripes (length $20 \mu \mathrm{m}$, height $20 \mathrm{~nm}$, width $2 \mu \mathrm{m}$, separated by $3.6 \mu \mathrm{m}$ ) has been obtained by electron beam lithography followed by a second evaporation step (see Methods and Supplementary Section A for further details). With this geometry, we find that the spin polarization of the photoexcited electrons is projected onto the sample plane with high efficiency compared with non-patterned devices exploiting optical spin injection at grazing incidence ${ }^{19}$. Indeed, we have calculated that the maximum $c_{y}$ value that can be obtained in a Ge sample covered with a uniform 4-nm-thick Pt layer is about one half of the maximum value obtained with the present set-up.

Pt has been chosen as the patterning material because, thanks to its high atomic number, it effectively functions as a spin detector. Spin accumulation can be revealed because it induces a spin current diffusing in $\mathrm{Ge}$ towards the $\mathrm{Pt} / \mathrm{Ge}$ interface ${ }^{19}$. Injection of a spin current density $\mathbf{J}_{\mathrm{s}}$ into the Pt layer gives origin to the inverse spin Hall effect (ISHE) via spin-dependent scattering with Pt nuclei (Fig. 1e), resulting in the generation of an electromotive field $\mathbf{E}_{\text {ISHE }}$ (refs 20,21)

$$
\mathbf{E}_{\mathrm{ISHE}}=D_{\mathrm{ISHE}} \mathbf{J}_{\mathrm{s}} \times \mathbf{u}_{\mathbf{P}}
$$

where $D_{\text {ISHE }}$ is a coefficient that represents the ISHE efficiency and $\mathbf{u}_{\mathbf{P}}$ is the unit vector corresponding to the direction of the spin polarization $\mathbf{P}$. We can then measure the electromotive force 


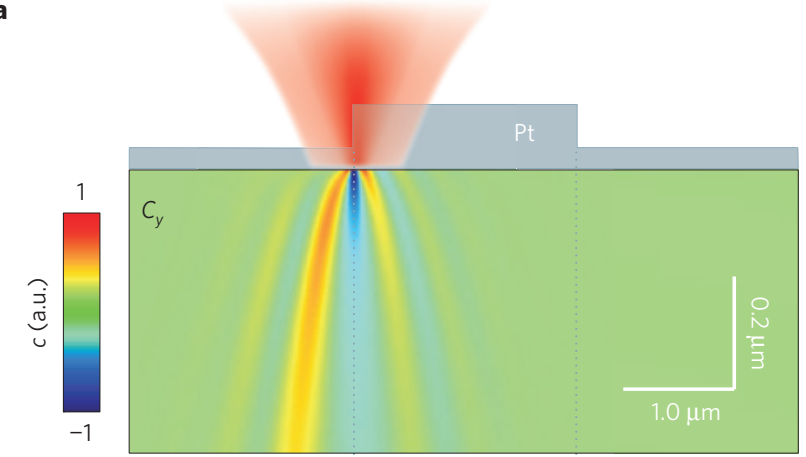

c

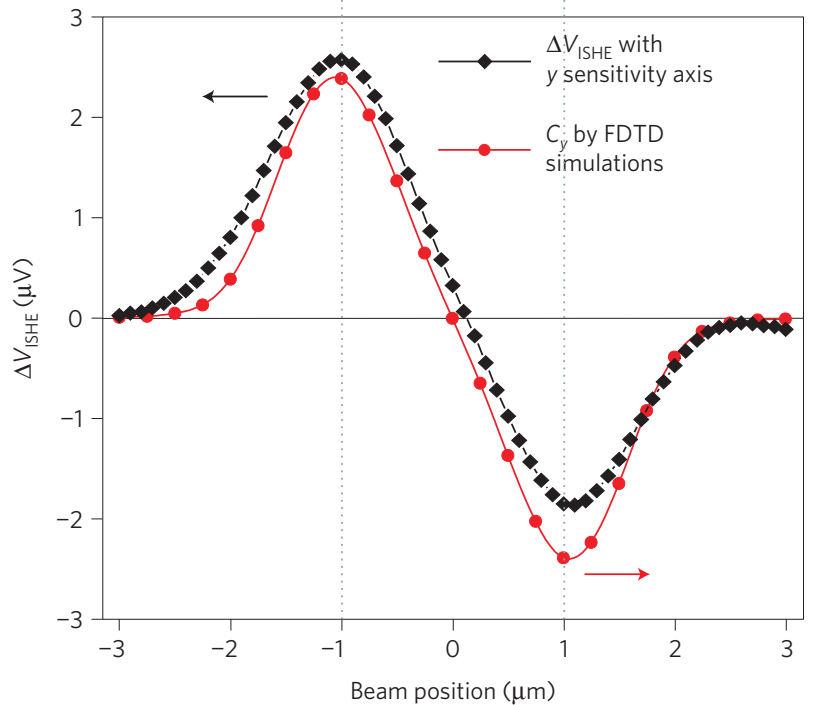

b

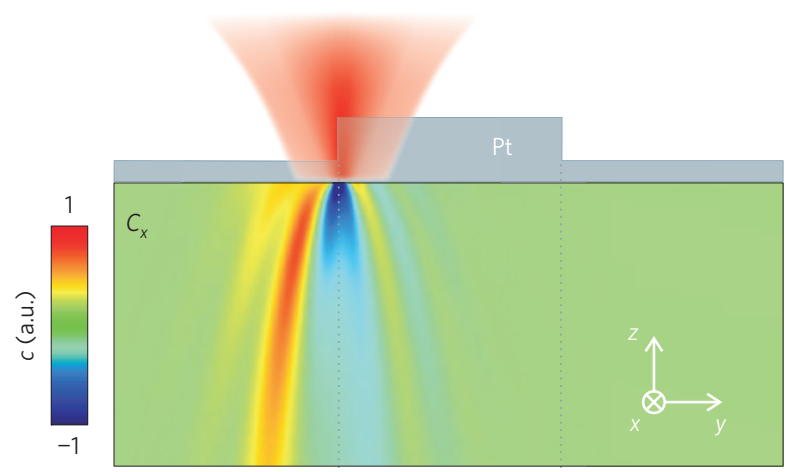

d

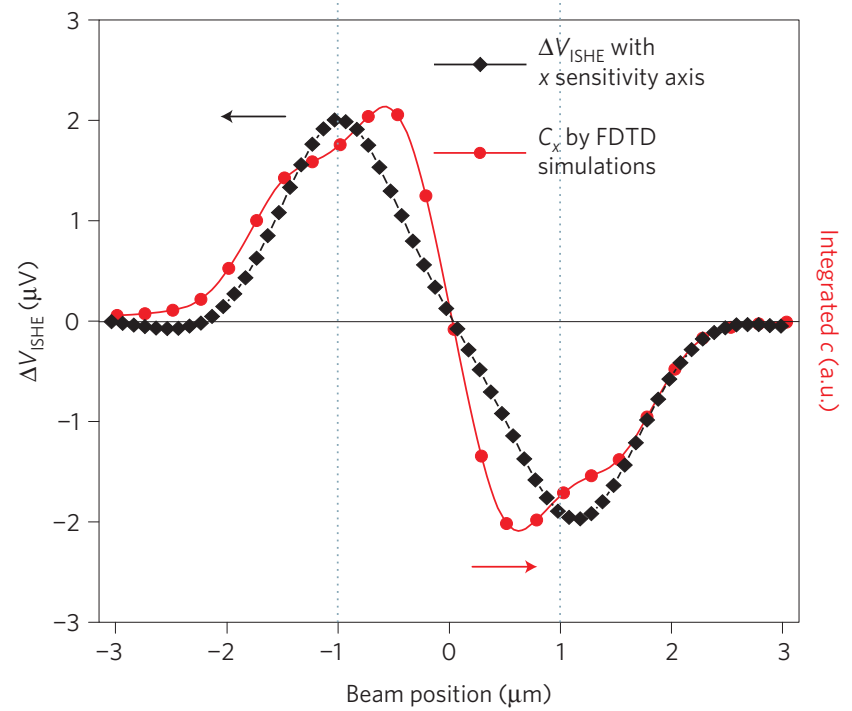

Figure $\mathbf{3}$ | Simulations and comparison with the experimental data. a,b, Finite-difference time-domain (FDTD) numerical simulations showing the map of $c_{y}(\mathbf{a})$ and $c_{x}(\mathbf{b})$ obtained when a Gaussian light beam ( $h v=0.8 \mathrm{eV}$, full-width at half-maximum $\left.w=1.5 \mu \mathrm{m}\right)$ is shone on one edge of the Pt stripe. Note that both $c_{y}$ and $c_{x}$ exhibit a net positive average value. The simulated region is $6 \times 6 \times 6 \mu \mathrm{m}^{3}$, with an adaptive mesh size down to $2 \mathrm{~nm}$ in the proximity of the stripe edges. c,d, Comparison between the $\Delta V_{\text {ISHE }}$ average profile obtained across a 2 - $\mu$ m-wide Pt stripe by setting the ISHE sensitivity axis perpendicular or parallel to the stripe axis (as in Fig. $2 \mathrm{~d}, \mathrm{f}$, respectively) and the corresponding calculated $c_{y}(\mathbf{c})$ or $c_{x}(\mathbf{d})$ normalized value integrated over the whole simulation region as a function of the beam position across the Pt stripe.

$\Delta V_{\mathrm{ISHE}} \approx E_{\mathrm{ISHE}} \cdot d$ generated between two Au contacts evaporated at the edges of the Pt layer (Fig. 1c,d), $d$ being the lateral size of the region from which electrons diffuse into the Pt overlayer (see Supplementary Section B). Owing to the position of the Au contacts (Fig. 1d), $\Delta V_{\text {ISHE }}$ is determined by the $\mathbf{E}_{\mathrm{ISHE}}$ component pointing along $x$. As $\mathbf{J}_{\mathrm{s}}$ is parallel to the $z$ direction, our set-up is thus sensitive to the $y$ component of $\mathbf{P}$.

By using uniform illumination, as shown in Fig. 1a, the opposite spin populations generated at the two edges of the stripe provide spin current densities that counterbalance each other, giving rise to a null $\Delta V_{\text {ISHE }}$. Therefore, to separately detect each spin population, one needs to break the illumination symmetry. A diffraction-limited confocal set-up (numerical aperture 0.7, giving a full-width at halfmaximum beam size $w \approx 1.5 \mu \mathrm{m}$ ) has been exploited to selectively generate the desired spin density at each edge of the Pt stripe (Fig. 1d). At the same time, the optical reflectivity signal of the set of Pt stripes has been monitored by an InGaAs detector (Fig. 2a,b). All measurements have been performed at room temperature.

The two-dimensional (2D) scan of Fig. 2c, performed with an incident light power $W=150 \mu \mathrm{W}$, clearly shows a strong $\Delta V_{\text {ISHE }}$ signal changing sign across the stripes. When the Gaussian beam illuminates one of the ridge edges along the $y$ axis, we observe a dramatic increase of the ISHE signal, which, in this geometry, is sensitive to the spin component perpendicular to the stripe long edge of photo-generated electrons entering the Pt layer. A reversed effect is observed on the opposite stripe edge. A similar result is obtained when the stripes are rotated with respect to the $\mathrm{Au}$ electrodes and the ISHE sensitivity axis is set parallel to the stripe edges (Fig. 2e). Asymmetries in the absolute value of the profiles have to be ascribed to photoelectric effects associated with a residual light intensity modulation. Such effects induce a variation of the instrumental background in correspondence with the stripes, which have a different reflectivity from that of the surrounding film (see Supplementary Section C). Slight aberrations of the light beam might also contribute to these asymmetries.

By applying a one-dimensional spin drift-diffusion model (see Supplementary Section B), and noting that, at $h v=E_{\mathrm{d}},|\mathbf{P}| \approx 50 \%$, we estimate that the majority of the electrons enter the Pt overlayer before depolarizing. This result stems from the light absorption length in $\mathrm{Ge}(\approx 2.2 \mu \mathrm{m}$ at $h v=0.8 \mathrm{eV})$ being comparable to the spin diffusion length $\Lambda_{\mathrm{Ge}} \approx 2.5 \mu \mathrm{m}$ of the conduction band electrons along the $\mathrm{L}$ states $^{8,19}$. Notably, the maximum absolute value of $\Delta V_{\text {ISHE }}\left(\Delta V_{\text {ISHE }}=2.4 \mu \mathrm{V}\right.$ at $\left.W=150 \mu \mathrm{W}\right)$, when properly normalized to account for the different experimental set-ups (see Supplementary Section B), is similar to the value measured on a device exploiting optical orientation at grazing incidence ${ }^{19}$. This gives an experimental confirmation of the efficiency and reliability of the present configuration. 
a
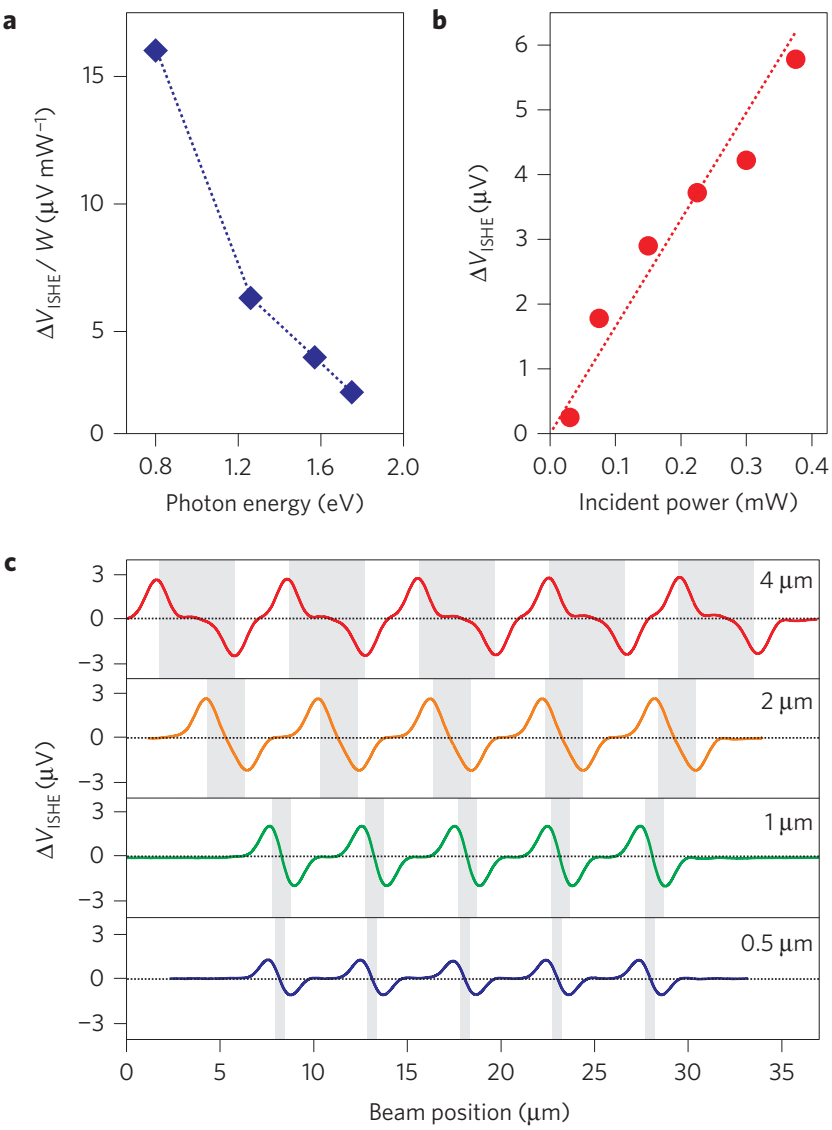

Figure 4 | Photon energy, excitation power and stripe width dependence of the ISHE signal. $\mathbf{a}, \Delta V_{\text {ISHE }}$ normalized by the incident power $W$, as a function of the incident photon energy $h v . \Delta V_{\text {ISHE }} / W$ is maximum when $h v=0.8 \mathrm{eV}$, in resonance with the Ge direct bandgap at room temperature, and follows the initial electron spin polarization curve discussed in the literature. $\mathbf{b}, \Delta V_{\text {ISHE }}$ as a function of the incident power. The dotted line represents a linear fit. $\mathbf{c}$, Dependence of the differential voltage signal $\Delta V_{\text {ISHE }}$ on the Pt stripe width for an incident photon energy $h v=0.8 \mathrm{eV}$ and $W=150 \mu \mathrm{W}$. The grey bands indicate the stripe position.

Numerical simulations allow us to correlate the experimental $\Delta V_{\mathrm{ISHE}}$ map with the in-plane spin density of photogenerated electrons in the Ge wafer. Figure 3a,b shows maps of $c_{y}$ and $c_{x}$ calculated when the light beam is centred on one of the edges of the Pt stripe. The resulting interference pattern indicates non-zero $c_{x}$ and $c_{y}$ values, which correspond to the detection of a net ISHE signal in the experimental geometry of Fig. 2c,e, respectively. Figure $3 c, d$ shows the comparison between the $\Delta V_{\text {ISHE }}$ measured with an ISHE sensitivity axis perpendicular or parallel to the Pt stripe and the calculated $c_{y}$ and $c_{x}$ values integrated over the Ge volume as the laser beam is moved across the stripe. The $\Delta V_{\text {ISHE }}$ and integrated $c_{y}$ and $c_{x}$ normalized profiles are in excellent agreement, demonstrating that the ISHE signal delivers an accurate mapping of the spin density.

To demonstrate the spin-related origin of the signal, we have repeated the above measurements, replacing the Pt overlayer with a $\mathrm{Cu}$ overlayer. A negligible ISHE voltage was obtained in this case (see Supplementary Section D), in agreement with the much smaller spin-dependent scattering occurring in $\mathrm{Cu}$ with respect to Pt. However, the ISHE signal is promptly retrieved when a Pt layer is inserted between the $\mathrm{Cu}$ layer and the $\mathrm{Cu}$ stripes (see Supplementary Section D), thus demonstrating the genuine origin of the detected signal. The angular dependence of the ISHE signal (see Supplementary Section E) was also investigated: the results are completely in line with our interpretation.
The measured voltage signal $\Delta V_{\text {ISHE }}$ depends on the incident photon energy. When the photon energy $h v$ is resonant with the Ge direct gap, only degenerate $\mathrm{HH}$ and $\mathrm{LH}$ states at $\Gamma$ participate in direct optical transitions to the conduction band. When $h v \approx E_{\mathrm{d}}+\Delta E_{\mathrm{SO}}$, SO states are also involved in the transitions and the initial electron spin polarization is reduced ${ }^{2,22}$. This trend is shown in Fig. $4 \mathrm{a}$, where $\Delta V_{\mathrm{ISHE}}$, normalized by the incident power $W$, is shown as a function of $h v . \Delta V_{\text {ISHE }} / W$ is maximum for $h v=0.8 \mathrm{eV}$ and becomes negligible when $h v=1.76 \mathrm{eV}$, in excellent agreement with theoretical calculations $\mathrm{s}^{22}$

Figure $4 \mathrm{~b}$ reveals instead that, up to $W=400 \mu \mathrm{W}$, the dependence of the ISHE signal, measured for $h v=0.8 \mathrm{eV}$, is linear with the number of initial spin-oriented electrons, ruling out a significant influence of sample heating, which, by increasing electron scattering, generally lowers the spin lifetime. Conversely, spin-related thermal effects such as those reported in refs 23,24 can be excluded, as they can manifest only in proximity to a ferromagnetic material.

We have also repeated the measurements by varying the stripe width from $4 \mu \mathrm{m}$ down to $500 \mathrm{~nm}$ (Fig. 4c). Interestingly, $\Delta V_{\mathrm{ISHE}}$ follows the same behaviour within the entire investigated width range, whereas the slight decrease of the signal dynamics when decreasing the stripe width can be rather attributed to the combined effect of the diffraction-limited spatial resolution of our setup and the smearing of the two complementary spin distributions as electrons diffuse towards the $\mathrm{Ge} / \mathrm{Pt}$ interface (see Supplementary Section B). This paves the way towards engineering an ordered array of complementary spin densities within the nanometric scale, as demonstrated for the stripe set with $500 \mathrm{~nm}$ width, even in confined geometries in both in-plane directions (see Supplementary Section E).

In conclusion, we have designed and experimentally tested a Ge-based opto-spintronic device that works as a spin photovoltaic generator that provides the simultaneous generation of two spatially separated populations with opposite spin projection in the plane of the device when circularly polarized light is absorbed. The optically induced spin voltage can be properly engineered by varying the stripe dimensions, while different combinations of semiconductor and patterned overlayer materials can be employed. In the proposed device it is possible to switch the sign of the pure spin photovoltage by reversing the circular polarization of the incoming light. Given that the low injection power prevents possible problems related to saturation, the operational frequency of the spin voltage reversal is limited by the modulation frequency of the light ellipticity. The simplicity of the proposed devices makes them appealing in a wide range of spintronics applications.

\section{Methods}

Set up. Continuous-wave laser diodes at $h v=0.8,1.26,1.57$ and $1.76 \mathrm{eV}$ were employed as light sources. The polarization of the light entering the confocal set-up was switched between two opposite circular states at a frequency $\omega=50 \mathrm{kHz}$ by means of a photo-elastic modulator (PEM). The ISHE signal between the two Au electrodes was then demodulated by a lock-in amplifier at the fundamental frequency. The signal demodulated at the second harmonic was also detected to monitor possible artefacts associated with residual light intensity modulation (see Supplementary Section C).

Sample. Pt films (nominal thickness $4 \mathrm{~nm}$ ) were deposited by electron-beam evaporation on a cleaned n-type (As-doped) Ge wafer $\left(N_{\mathrm{d}}=2 \times 10^{16} \mathrm{~cm}^{3}\right)$. Stripe of Pt $20 \mathrm{~nm}$ in thickness with variable widths (500 nm, $1 \mu \mathrm{m}, 2 \mu \mathrm{m}$ and $4 \mu \mathrm{m})$ and identical lengths $(20 \mu \mathrm{m})$, as well as discs $450 \mathrm{~nm}$ in diameter (see Supplementary Section A) were fabricated using a positive pattern process by electron-beam lithography. A poly(methyl methacrylate) resist was exposed to a $300 \mu \mathrm{C} \mathrm{cm}^{-2}$ dose at $30 \mathrm{keV}$. After the development process obtained by a solution of methyl-isobutyl-ketone and isopropanol (1:3) for $90 \mathrm{~s}$ at room temperature, $20 \mathrm{~nm}$ of Pt (or Cu, see Supplementary Section D) was evaporated to create the metallic stripe- and disc-shaped discontinuities. Gold pads (nominal thickness $100 \mathrm{~nm}$, width $600 \mu \mathrm{m}$, length $5 \mathrm{~mm}$, spacing $5 \mathrm{~mm}$ ) were then evaporated on the Pt (or $\mathrm{Cu}$ ) film. To improve gold adhesion, an extra 3-nm-thick Ti layer was evaporated before gold deposition. 
Numerical simulations. Full vectorial three-dimensional electromagnetic simulations were performed by the finite-difference time-domain (FDTD) approach, employing commercial software (FDTD Solutions, version 8.5.3, Lumerical Inc). The dielectric functions of Pt and Ge have been obtained from refs 25,26 , respectively. The simulated region was $6 \times 6 \times 6 \mu \mathrm{m}^{3}$, with an adaptive mesh size down to $2 \mathrm{~nm}$ in proximity to the stripe edges. For the simulation of the line scan (Fig. 3b), $c_{x}$ and $c_{y}$ signals have been integrated inside the Ge film all over the simulation volume as a function of the scanning Gaussian beam position.

\section{Received 6 February 2014; accepted 23 May 2014;} published online 22 June 2014

\section{References}

1. Zutić, I., Fabian, J. \& Das Sarma, S. Spintronics: Fundamentals and applications. Rev. Mod. Phys. 76, 323-410 (2004).

2. Meier, F. \& Zakharchenya, B. P. Optical Orientation: Modern Problems in Condensed Matter Sciences Vol. 8 (Elsevier Science, 1984).

3. Jansen, R. Spintronics: Solar spin devices see the light. Nature Mater. 12, 779-780 (2013).

4. Endres, B. et al. Demonstration of the spin solar cell and spin photodiode effect. Nature Commun. 4, 2068 (2013).

5. Kondo, T., Hayafuji, J-J. \& Munekata, H. Investigation of spin voltaic effect in a p-n heterojunction. Jpn. J. Appl. Phys. 45, L663 (2006).

6. Zutić, I., Fabian, J. \& Das Sarma, S. Spin-polarized transport in inhomogeneous magnetic semiconductors: Theory of magnetic/nonmagnetic $\mathrm{p}-\mathrm{n}$ junctions. Phys. Rev. Lett. 88, 066603 (2002).

7. Zutić, I., Fabian, J. \& Das Sarma, S. Spin injection through the depletion layer: A theory of spin-polarized $\mathrm{p}-\mathrm{n}$ junctions and solar cells. Phys. Rev. B 64, 121201(R) (2001).

8. Guite, C. \& Venkataraman, V. Temperature dependence of spin lifetime of conduction electrons in bulk germanium. Appl. Phys. Lett. 101, 252404 (2012).

9. Pezzoli, F. et al. Optical spin injection and spin lifetime in Ge heterostructures. Phys. Rev. Lett. 108, 156603 (2012).

10. Hochberg, M. \& Baehr-Jones, T. Towards fabless silicon photonics. Nature Photon. 4, 492-494 (2010).

11. Süess, M. J. et al. Analysis of enhanced light emission from highly strained germanium microbridges. Nature Photon. 7, 466-472 (2013).

12. Allenspach, R., Meier, F. \& Pescia, D. Experimental symmetry analysis of electronic states by spin-dependent photoemission. Phys. Rev. Lett. 51, 2148-2150 (1983).

13. Ferrari, A., Bottegoni, F., Isella, G., Cecchi, S. \& Ciccacci, F. Epitaxial $\mathrm{Si}_{1-x} \mathrm{Ge}_{x}$ alloys studied by spin-polarized photoemission. Phys. Rev. B 88, 115209 (2013).

14. Lampel, G. Nuclear dynamic polarization by optical electronic saturation and optical pumping in semiconductors. Phys. Rev. Lett. 20, 491-493 (1968).

15. Loren, E. J. et al. Hole spin relaxation and intervalley electron scattering in germanium. Phys. Rev. B 84, 214307 (2011).
16. Sinova, J. \& Zutić, I. New moves of the spintronics tango. Nature Mater. 11, 368-371 (2012).

17. Jungwirth, T., Wunderlich, J. \& Olejník, K. Spin Hall effect devices. Nature Mater. 11, 382-390 (2012).

18. McMaster, W. H. Matrix representation of polarization. Rev. Mod. Phys. 33, 8-27 (1961).

19. Bottegoni, F. et al. Photoinduced inverse spin Hall effect in $\mathrm{Pt} / \mathrm{Ge}(001)$ at room temperature. Appl. Phys. Lett. 102, 152411 (2013).

20. Saitoh, E., Ueda, M., Miyajima, H. \& Tatara, G. Conversion of spin current into charge current at room temperature: Inverse spin-Hall effect. Appl. Phys. Lett. 88, 182509 (2006).

21. Valenzuela, S. O. \& Tinkham, M. Direct electronic measurement of the spin Hall effect. Nature 442, 176-179 (2006).

22. Rioux, J. \& Sipe, J. E. Optical injection and control in germanium: Thirty-band $k \cdot p$ theory. Phys. Rev. B 81, 155215 (2010).

23. Slachter, A., Bakker, F. L., Adam, J-P. \& van Wees, B. J. Thermally driven spin injection from a ferromagnet into a non-magnetic metal. Nature Phys. 6, 879-882 (2010).

24. Le Breton, J-C. et al. Thermal spin current from a ferromagnet to silicon by Seebeck spin tunnelling. Nature 475, 82-85 (2011).

25. Palik, D. (ed.) Handbook of Optical Constants of Solids III (Academic, 1998).

26. Adachi, S. Model dielectric constants of Si and Ge. Phys. Rev. B 38, 12966-12976 (1988)

\section{Acknowledgements}

The authors would like to thank L. Duò for fruitful discussions. The CARIPLO foundation is acknowledged for partially funding this work through the NANOGAP (2010-0632) project. The research leading to these results has received funding from the European Union's Seventh Framework Programme under grant agreement No 613055.

\section{Author contributions}

M.B. and G.I. fabricated the samples. F.B. and M.C. carried out the measurements. P.B. performed the numerical simulations. M.C., M.F. and F.C. provided the confocal apparatus with light polarization analysis. F.B. and M.C. performed the data analysis. F.C., G.I. and M.F. coordinated the entire work. All the authors contributed to the writing of the manuscript.

\section{Additional information}

Supplementary information is available in the online version of the paper. Correspondence and requests for materials should be addressed to M.F.

\section{Competing financial interests}

The authors declare no competing financial interests. 